\title{
A CONSTRAINT ON THE EXISTENCE OF SIMPLE TORSION-FREE LIE MODULES
}

\author{
DANIEL BRITTEN, FRANK LEMIRE, AND VAHID TAROKH
}

(Communicated by Maurice Auslander)

\begin{abstract}
For any simple Lie algebra $L$ with Cartan subalgebra $H$ the classification of all simple $H$-diagonalizable $L$-modules having a finite-dimensional weight space is known to depend on determining the simple torsion-free $L$ modules of finite degree. It is further known that the only simple Lie algebras which admit simple torsion-free modules of finite degree are those of types $A_{n}$ and $C_{n}$. For the case of $A_{n}$ we show that there are no simple torsion-free $A_{n}$ modules of degree $k$ for $n \geq 4$ and $2 \leq k \leq n-2$. We conclude with some examples showing that there exist simple torsion-free $A_{n}$-modules of degrees $1, n-1$, and $n$.
\end{abstract}

Let $L$ be a finite-dimensional simple Lie algebra over the complex numbers $\mathbb{C}$, and $H$ be a fixed Cartan subalgebra of $L$. An $H$-diagonalizable $L$-module $M$ is said to be: of degree $k$ provided each weight space of $M$ is of dimension $k$; torsion-free provided each root vector of $L$ acts on each weight space of $M$ in a one-to-one manner; and pointed provided it is simple and has a 1dimensional weight space.

Theorem 4.18 of [F] describes simple $H$-diagonalizable $L$-modules having a finite-dimensional weight space as the amalgam of highest weight modules and torsion-free modules, thereby reducing the classification of such modules to determining the simple torsion-free modules of degree $k<\infty$. The complete classification of pointed modules was presented in [BL1]. In studying modules of minimal weight space dimension greater than 1 , we must study simple torsion-free modules of degree greater than 1. In [F], Fernando showed that only $A_{n}, n \geq 1$, and $C_{n}, n \geq 2$, possess torsion-free modules. It is easy to see that no simple torsion-free modules of minimal weight space dimension greater than 1 exist for $A_{1}$. The existence of simple torsion-free $A_{n}$-modules of arbitrary degree for $n=2,3$ has been established in [BBL].

In this note, we further restrict the existence of torsion-free $A_{n}$-modules by proving

Main Theorem. There are no simple torsion-free $A_{n}$-modules of degree $k$ for $n \geq 4$ and $2 \leq k \leq n-2$.

Received by the editors October 30, 1991 and, in revised form, November 11, 1993.

1991 Mathematics Subject Classification. Primary 17B10.

The first author was supported in part by NSERC Grant \#A8471.

The second author was supported in part by NSERC Grant \#A7742.

(C)1995 American Mathematical Society 
We consider $A_{n}$ to be the $(n+1) \times(n+1)$ traceless matrices over $\mathbb{C}$. Let $\left\{E_{i j} \mid 1 \leq i, j \leq n+1\right\}$ be a standard set of matrix units and $\left\{\varepsilon_{i} \mid 1 \leq i \leq n+1\right\}$ be a standard basis of $\mathbb{C}^{n+1}$. Naturally, we think of $\Delta=\left\{\alpha_{i}=\varepsilon_{i}-\varepsilon_{i+1} \mid 1 \leq i \leq n\right\}$ as a base of the root system $\Phi=\left\{ \pm\left(\varepsilon_{i}-\varepsilon_{j}\right) \mid 1 \leq i<j \leq n\right\}$ of $A_{n}$. If $i \leq j$ and $\alpha=\alpha_{i}+\cdots+\alpha_{j}=\varepsilon_{i}-\varepsilon_{j+1}$, then the root vectors $X_{\alpha}, X_{-\alpha}$ are $E_{i, j+1}, E_{j+1, i}$, respectively, and $h_{\alpha}=E_{i i}-E_{j+1, j+1} . \quad H=\operatorname{span}_{\mathbb{C}}\left\{h_{\alpha} \mid \alpha \in \Delta\right\}$ is our fixed Cartan subalgebra. In subsequent sections we will have occasion to use other simple bases for the root system $\Phi$ of $A_{n}$. In all cases we order the simple basis elements to preserve the connectedness of adjacent roots.

Let $U=U\left(A_{n}\right)$ be the universal enveloping algebra of $A_{n}$ and $U_{0}=$ $U_{0}\left(A_{n}\right)=\{u \in U \mid[u, h]=0, \forall h \in H\}$. Using Theorem 2.1 of [BL2], the elements $u \in U_{0}$ can be characterized as linear combinations of products of $h_{\alpha}, \alpha \in \Delta$, and elements of the form

$$
u=E_{i_{1}, i_{2}} \cdots E_{i_{l-1}, i_{l}} E_{i_{l}, i_{1}}
$$

where $i_{1}, \ldots, i_{l}$ are distinct in $\{1, \ldots, n+1\}$.

In the torsion-free setting that we are working in it is easy to see that $M$ is a simple $U$-module if and only if for any weight $\Theta M_{\Theta}$ is a simple $U_{0}$-module with $M=U M_{\Theta}$. This means that we can restrict our attention to an arbitrary weight space $M_{\Theta}$ and so, at our convenience, we can choose $\theta$ such that it satisfies the General Assumption below.

The Main Theorem is proved by a sequence of lemmas under the following

General Assumption. $M$ is a torsion-free $A_{n}$-module of degree $k<\infty$ and $n \geq 2$. Using the torsion-free assumption we fix a weight $\boldsymbol{\theta}$ of $M$ such that $\boldsymbol{\Theta}\left(\bar{h}_{\alpha}\right) \neq 0$ for all $\alpha \in \Phi$. Denote the restriction of $X_{-\alpha} X_{\alpha}$ to the weight space $M_{\Theta}$ by $\mathscr{A}_{\alpha}$.

Let $i, j, m$ be any three distinct values in $\{1, \ldots, n+1\}$. Set

$$
\begin{aligned}
& c_{1}(i, j, m)=E_{j i} E_{i j}, \quad c_{2}(i, j, m)=E_{m j} E_{j m}, \quad c_{3}(i, j, m)=E_{m i} E_{i m}, \\
& c_{4}(i, j, m)=E_{m i} E_{i j} E_{j m}, \quad c_{5}(i, j, m)=E_{m j} E_{j i} E_{i m}, \\
& h_{1}(i, j, m)=E_{i i}-E_{j j}, \quad \text { and } h_{2}(i, j, m)=E_{j j}-E_{m m} .
\end{aligned}
$$

Drop $(i, j, m)$ to simplify notation. One can show that the following identities hold in $U$ :

$$
\begin{aligned}
{\left[c_{1}, c_{2}\right] } & =\left[c_{2}, c_{3}\right]=-\left[c_{1}, c_{3}\right]=c_{5}-c_{4} \\
{\left[c_{1}, c_{4}\right] } & =-c_{2} c_{1}+c_{3} c_{1}-c_{4} h_{1}+c_{3} h_{1}-c_{5}+c_{4} \\
{\left[c_{2}, c_{4}\right] } & =c_{2} c_{1}-c_{3} c_{2}-c_{4} h_{2}+c_{5}-c_{4} \\
{\left[c_{1},\left[c_{1}, c_{2}\right]\right] } & =2 c_{2} c_{1}-2 c_{3} c_{1}+\left(c_{5}+c_{4}\right) h_{1}-2 c_{3} h_{1}+2\left[c_{1}, c_{2}\right]
\end{aligned}
$$

Let $\tilde{c}_{i}$ denote the action of $c_{i}$ on $M_{\Theta}$ and $\tilde{h}_{i}=\boldsymbol{\Theta}\left(h_{i}\right)$. We use $W \leq M_{\boldsymbol{\Theta}}$ to indicate that $W$ is a subspace of $M_{\Theta}$.

Lemma 6. If $W \leq M_{\Theta}$ is invariant under $\mathscr{A}_{\alpha}$ for all $\alpha \in \Phi$, then $W$ is $a$ $U_{0}$-submodule of $M_{\Theta}$.

Proof. It suffices to show that $W$ is invariant under all $u$ of form (1). By hypothesis, this is true if $l=2$. Assume $l=3$ so that $u=c_{4}(i, j, m)=$ $E_{m i} E_{i j} E_{j m}$. Since $\boldsymbol{\Theta}\left(h_{1}\right) \neq 0$, we can use (2) and (5) to solve for $\tilde{c}_{4}$ and $\tilde{c}_{5}$ 
and see that $\tilde{c}_{4}$ and $\tilde{c}_{5}$ leave $W$ invariant. Assume that $W$ is invariant under all elements of form (1) with $l<m$, and observe that in $U_{0}$

$$
\begin{aligned}
& \left(E_{i_{1}, i_{2}} \cdots E_{i_{m-1} i_{m}} E_{i_{m}, i_{1}}\right)\left(E_{i_{m-1}, i_{1}} E_{i_{1}, i_{m-1}}\right) \\
& \quad=\left(E_{i_{1}, i_{2}} \cdots E_{i_{m-2}, i_{m-1}} E_{i_{m-1}, i_{1}}\right)\left(E_{i_{m-1}, i_{m}} E_{i_{m}, i_{1}} E_{i_{1}, i_{m-1}}\right) .
\end{aligned}
$$

Since the two expressions of form (1) on the right-hand side of (10) leave $W$ invariant as does the invertible operator $E_{i_{m-1}, i_{1}} E_{i_{1}, i_{m-1}}$, the result follows by induction.

Notation. For each $1 \leq m \leq n$, let $\Phi^{m}$ be the root subsystem generated by $\Delta^{m}=\left\{\alpha_{m}, \ldots, \alpha_{n}\right\} \subseteq \Delta$. If $\alpha, \beta, \alpha+\beta \in \Phi$, then $A(\alpha, \beta)$ denotes the $A_{2}$ subalgebra of $A_{n}$ generated by $X_{ \pm \alpha}$ and $X_{ \pm \beta}$.

If $\mathscr{A}_{\alpha}$ acts like a complex multiple of the identity map on $W \leq M_{\Theta}$, then we say that $\mathscr{A}_{\alpha}$ is a scalar on $W$.

Lemma 8. Let $\alpha, \beta, \alpha+\beta \in \Phi$ and $W \leq M_{\boldsymbol{\Theta}}$. Then

(i) if $\mathscr{A}_{\alpha}$ is a scalar on $W$ and $W$ is invariant under $\mathscr{A}_{\beta}$, then $W$ is a $U_{0}(A(\alpha, \beta))$-module,

(ii) if $\mathscr{A}_{\alpha}$ and $\mathscr{A}_{\beta}$ are both scalars on $W$, then all elements in $U_{0}(A(\alpha, \beta))$ are scalars on $W$, and

(iii) if $\mathscr{A}_{\alpha}$ is a scalar on $W$ and $\mathscr{A}_{\beta}$ is a scalar on $W$ for all $\beta \in \Phi$ such that $\alpha+\beta \in \Phi$, then $\mathscr{A}_{\gamma}$ is a scalar on $W$ for all $\gamma \in \Phi$.

Proof. Let $w \in W$ and $\mathscr{A}_{\alpha}$ be a scalar on $W$. Without loss of generality, we may assume that $X_{\alpha}=E_{i j}$ and $X_{\beta}=E_{j m}$. Then with $\tilde{c}_{1}=\mathscr{A}_{\alpha}$ and $\tilde{c}_{2}=\mathscr{A}_{\beta}$ we have that $0=\left[\tilde{c}_{1}, \tilde{c}_{2}\right] w=\left(\tilde{c}_{5}-\tilde{c}_{4}\right) w$ and so, by $(2),\left[\tilde{c}_{r}, \tilde{c}_{s}\right] w=0$ for $1 \leq r, s \leq 3$. By (2) and (5), $\tilde{c}_{4}$ and $\tilde{c}_{5}$ are expressible in terms of $\tilde{c}_{1}, \tilde{c}_{2}, \tilde{c}_{3}$. Therefore,

$$
\begin{aligned}
& 0=\left[\tilde{c}_{1}, \tilde{c}_{4}\right] w=\left(-\tilde{c}_{2} \tilde{c}_{1}+\tilde{c}_{3} \tilde{c}_{1}-\tilde{c}_{4} \tilde{h}_{1}+\tilde{c}_{3} \tilde{h}_{1}\right) w \\
& 0=\left[\tilde{c}_{2}, \tilde{c}_{4}\right] w=\left(\tilde{c}_{2} \tilde{c}_{1}-\tilde{c}_{3} \tilde{c}_{2}-\tilde{c}_{4} \tilde{h}_{2}\right) w
\end{aligned}
$$

and we have

$$
\tilde{c}_{2} \tilde{c}_{1}\left(\tilde{h}_{1}+\tilde{h}_{2}\right) w=\tilde{c}_{3}\left(\tilde{c}_{1} \tilde{h}_{2}+\tilde{c}_{2} \tilde{h}_{1}+\tilde{h}_{1} \tilde{h}_{2}\right) w
$$

Since, if $w \neq 0$, the left-hand side of equality (11) is a nonzero element in $W$, $\left(\tilde{c}_{1} \tilde{h}_{2}+\tilde{c}_{2} \tilde{h}_{1}+\tilde{h}_{1} \tilde{h}_{2}\right)$ is an invertible operator on $W$ and we see that $\tilde{c}_{3}$ leaves $W$ invariant, (i) follows from this and Lemma 6 . Also, if $\mathscr{A}_{\beta}$ is a scalar on $W$, then (11) implies $\tilde{c}_{3}$ is a scalar on $W$ and (10) implies that $\tilde{c}_{4}$ is and (ii) follows.

Now for (iii), without loss of generality, take $\alpha_{1}=\alpha$ in $\Delta$. Clearly, $\mathscr{A}_{\alpha_{2}}$ and $\mathscr{A}_{-\left(\alpha_{1}+\alpha_{2}+\alpha_{3}\right)}$ are scalars on $W$. Part (ii) implies $\mathscr{A}_{\alpha_{1}+\alpha_{2}}$ is a scalar on $W$. $\mathscr{A}_{\alpha_{3}}$ is a scalar on $W$ since it denotes the action of an element of

$$
U_{0}\left(A\left(\alpha_{1}+\alpha_{2},-\left(\alpha_{1}+\alpha_{2}+\alpha_{3}\right)\right)\right)
$$

Continuing in this fashion one gets that $\mathscr{A}_{\alpha_{i}}$ is a scalar on $W$ for all $\alpha_{i} \in \Delta$, and the result follows from part (ii). 
Lemma 12. Fix an eigenvalue $\lambda_{i}$ of $\mathscr{A}_{\alpha_{i}}$ for each $\alpha_{i} \in \Delta$, and let $W_{i}$ be the corresponding eigenspace. For each $1 \leq j \leq n$, define $W^{(j-1)}=W_{1} \cap \cdots \cap W_{j-1}$. If $\mathscr{A}_{\alpha_{j}}$ is a scalar on $W^{(j-1)} \neq 0$ and $k>1$, then $M$ is not simple. (In particular, if for some $\beta \in \Phi \mathscr{A}_{\beta}$ is a scalar on $M_{\Theta}$, then $M$ is not simple.)

Proof. Assume that $M$ is simple of degree $k \geq 2 . W^{(j-1)} \neq 0$ is invariant under $\mathscr{A}_{\alpha}$ for each $\alpha \in \Phi^{j+1}$ because $\mathscr{A}_{\alpha} \mathscr{A}_{\alpha_{i}}=\mathscr{A}_{\alpha_{i}} \mathscr{A}_{\alpha}$ for $1 \leq i \leq j-1$. Lemma 8(i) implies $W^{(j-1)}$ is invariant under $\mathscr{A}_{\alpha}$ for $\alpha \in \Phi^{j}$ and in turn for $\alpha \in \Phi^{j-1}, \alpha \in \Phi^{j-2}, \ldots, \alpha \in \Phi^{1}=\Phi$. If $W^{(j-1)} \neq M_{\Theta}$, then by Lemma 6 , $W^{(j-1)}$ is a proper $U_{0}$-submodule of $M_{\Theta}$, which implies that $M$ is not simple.

If $W^{(j-1)}=M_{\Theta}$, then let $l$ be the smallest subscript such that $\mathscr{A}_{\alpha_{l}}$ is not a scalar on $M_{\Theta}$. By Lemma 6 and Lemma 8(ii), such an $l$ must exist. Note that $\Delta^{\prime}=\left\{\alpha_{1}^{\prime}=\alpha_{l}, \alpha_{2}^{\prime}=\alpha_{l-1}, \ldots, \alpha_{l}^{\prime}=\alpha_{1}, \alpha_{l+1}^{\prime}=-\left(\alpha_{l+1}+\alpha_{l}+\cdots+\alpha_{1}\right), \alpha_{l+2}^{\prime}=\right.$ $\left.-\alpha_{l+2}, \ldots, \alpha_{n}=-\alpha_{n}\right\}$ is a base such that if we let $W_{1}^{\prime}$ be an eigenspace corresponding to $\mathscr{A}_{\alpha_{1}^{\prime}}$, then $\mathscr{A}_{\alpha_{2}^{\prime}}$ is a scalar on $M_{\boldsymbol{\theta}} \neq W^{(1)^{\prime}}=W_{1}^{\prime} \neq 0$. We are now back to the case handled above.

Lemma 13. Let $n \geq 4$. If the degree $k$ of $M$ satisfies $2 \leq k \leq n-2$ and $v$ is an eigenvector of $\mathscr{A}_{\alpha}$ for each $\alpha \in \Delta^{3}$, then $M$ is not simple.

Proof. Suppose that $M$ is simple, and for $3 \leq i \leq n$ let $\lambda_{i}$ be the eigenvalue of $\mathscr{A}_{\alpha_{i}}$ belonging to $v$. Let $W_{i}$ be the eigenspace of $\mathscr{A}_{\alpha_{i}}$ belonging to $\lambda_{i}$. By Lemma 12, $\operatorname{dim} W_{i}<k \leq n-2$. Since we can complete $\left\{\alpha_{1}^{\prime}=\alpha_{3}, \ldots, \alpha_{n-2}^{\prime}=\right.$ $\left.\alpha_{n}\right\}$ to a base $\Delta^{\prime}$ for $\Phi$ and apply Lemma 12 to get in turn $\operatorname{dim}\left(W_{3} \cap W_{4}\right)<$ $n-3, \operatorname{dim}\left(W_{3} \cap W_{4} \cap W_{5}\right)<n-4, \ldots, \operatorname{dim}\left(W_{3} \cap \cdots \cap W_{n}\right)<n-(n-1)=1$. But by assumption, $v \in W_{3} \cap \cdots \cap W_{n}$. This contradiction gives the result.

Corollary 14. If $n \geq 4$ and $M$ is simple, then for $\alpha \in \Phi$ the eigenspace of $\mathscr{A}_{\alpha}$ are all of dimension greater than 1 .

Proof. If $W=\mathbb{C} v$ is a 1-dimensional eigenspace of $\mathscr{A}_{\alpha}$, then take $\alpha_{1}=\alpha$ in base $\Delta$ and note that $\mathscr{A}_{\alpha_{3}}, \ldots, \mathscr{A}_{\alpha_{n}}$ all have $v$ as an eigenvector. Lemma 13 implies that $M$ is not simple.

Proof of Main Theorem. Assume that $M$ is simple torsion-free of degree $k \leq$ $n-2$ and $n \geq 4$. If $k=2$, then we have the contradiction concerning the dimension of an eigenspace of $\mathscr{A}_{\alpha}$ given to us by Lemma 12 and Corollary 14 . Hence, $k \geq 3$.

This proves that the result is true for $n=4$ and begins our inductive proof. Now, assume that the result is true for all $m, 4 \leq m<n$, and $n \geq 5$.

As our first step, we establish the claim that, when $n \geq 5, k=n-2$ and each operator $\mathscr{A}_{\alpha}$ has a unique eigenvalue whose eigenspace has dimension $d=n-3$. For $n=5$, Lemma 12 and Corollary 14 imply that the claim is true. Continuing, we assume that $n \geq 6$. Let $V$ be either an eigenspace or a generalized eigenspace of $\mathscr{A}_{\alpha}$. By Corollary 14, $\operatorname{dim} V \geq 2$. Suppose that $2 \leq \operatorname{dim} V \leq n-4$. Take a base $\Delta=\left\{\alpha=\alpha_{1}, \ldots, \alpha_{n}\right\}$. Let $A_{m}$ be the subalgebra generated by the root vectors in $\Phi^{3} . U\left(A_{m}\right) V$ is a torsion-free $A_{m}$-module, and since $m=n-2 \geq 4$, the induction assumption says that there is a 1-dimensional $U_{0}\left(A_{m}\right)$-submodule of $V$, contrary to Lemma 13 . If $\mathscr{A}_{\alpha}$ has two distinct eigenvalues with corresponding dimensions $d_{1}$ and $d_{2}$, then $k \geq d_{1}+d_{2}>2 n-8$ and so we have arrived at the contradiction that $2 n-8<k \leq n-2$ or $n<6$. Hence, the claim is true. 
Our second claim is that if $n \geq 5$ and $W_{i}$ is the unique eigenspace of $\mathscr{A}_{\alpha_{i}}$ of dimension $n-3$ for $\alpha_{i} \in \Delta$, then $\operatorname{dim}\left(W_{i_{1}} \cap \cdots \cap W_{i_{j}}\right) \geq n-2-j$. This is known for $j=1$. Inductively assume that $\operatorname{dim}\left(W_{i_{1}} \cap \cdots \cap W_{i_{j-1}}\right) \geq n-2-(j-1)=$ $n-1-j$. Then

$$
\begin{aligned}
n-2 & \geq \operatorname{dim}\left(W_{i_{1}} \cap \cdots \cap W_{i_{j-1}}+W_{i_{j}}\right) \\
& =\operatorname{dim}\left(W_{i_{1}} \cap \cdots \cap W_{i_{j-1}}\right)+\operatorname{dim}\left(W_{i_{j}}\right)-\operatorname{dim}\left(W_{i_{1}} \cap \cdots \cap W_{i_{j}}\right) \\
& \geq n-1-j+n-3-\operatorname{dim}\left(W_{i_{1}} \cap \cdots \cap W_{i_{j}}\right) .
\end{aligned}
$$

Thus $\operatorname{dim}\left(W_{i_{1}} \cap \cdots \cap W_{i_{j}}\right) \geq n-2-j$.

When $j=n-3, \operatorname{dim}\left(W_{i_{1}} \cap \cdots \cap W_{i_{j}}\right) \geq 1$. If $W_{1} \cap \cdots \cap W_{j-1}=W_{1} \cap \cdots \cap$ $W_{j}$, then $\mathscr{A}_{\alpha_{j}}$ is a scalar on $W=W_{1} \cap \cdots \cap W_{j-1}$ and $M$ is not simple by Lemma 13. Therefore, $W_{1} \supset W_{1} \cap W_{2} \supset \cdots \supset W_{1} \cap \cdots \cap W_{n-3} \neq\{0\}$ and so $\operatorname{dim}\left(W_{1} \cap \cdots \cap W_{n-4}\right)=2$ and $\operatorname{dim}\left(W_{1} \cap \cdots \cap W_{n-3}\right)=1$. If, for some base $\Delta, \operatorname{dim}\left(W_{1} \cap \cdots \cap W_{n-4} \cap W_{n}\right)=1$, then since $\operatorname{dim}\left(W_{1} \cap \cdots \cap W_{n-3}\right)=1$ and $A_{\alpha_{n}}$ leaves $W_{1} \cap \cdots \cap W_{n-3}$ invariant, we have $W_{1} \cap \cdots \cap W_{n-3} \subset W_{n}$ and so $W_{1} \cap \cdots \cap W_{n-3}=W_{1} \cap \cdots \cap W_{n-4} \cap W_{n}$. This implies $W_{1} \cap \cdots \cap W_{n-3} \subset W_{n-2}$ and $\mathscr{A}_{\alpha_{n-2}}$ is a scalar on $W_{1} \cap \cdots \cap W_{n-3}$, contrary to Lemma 12 . Hence, we assume that for all bases $W_{1} \cap \cdots \cap W_{n-4} \subset W_{n}$. Consider the bases

$$
\Delta^{\prime}=\left\{\alpha_{1} \ldots, \alpha_{n-3}, \alpha_{n-2}+\alpha_{n-1},-\alpha_{n-1}, \alpha_{n-1}+\alpha_{n}\right\}
$$

and

$$
\Delta^{\prime \prime}=\left\{\alpha_{1} \ldots, \alpha_{n-4}, \alpha_{n-3}+\alpha_{n-2}+\alpha_{n-1},-\alpha_{n-1},-\alpha_{n-2}, \alpha_{n-2}+\alpha_{n-1}+\alpha_{n}\right\} .
$$

Since $\mathscr{A}_{\alpha_{n-2}+\alpha_{n-1}+\alpha_{n}}$ and $\mathscr{A}_{-\left(\alpha_{n-1}+\alpha_{n}\right)}$ are scalars on $W_{1} \cap \cdots \cap W_{n-4}$, Lemma 8(ii) says that $\mathscr{A}_{\alpha_{n-2}}$ is a scalar on $W_{1} \cap \cdots \cap W_{n-3} \subset W_{1} \cap \cdots \cap W_{n-4}$, and we again have a contradiction to Lemma 12.

Example. We provide examples of simple torsion-free $A_{n}$-modules of degrees $1, n-1$, and $n$. To this end we first observe that the algebra $\operatorname{gl}(n+1, \mathbb{C})$ can be identified with an operator algebra as follows. Let $\left\{x_{1}, \ldots, x_{n+1}\right\}$ denote a set of commuting variables, and set $X_{i}$ (respectively, $\partial_{i}$ ) to be multiplication (respectively, partial differentiation) by the variable $x_{i}$. Then the map which identifies the standard basis matrices $E_{i j}$ with the operator $X_{i} \partial_{j}$ is a Lie algebra isomorphism. The restriction of this map to the subalgebra $A_{n}$ of $n+1 \times n+1$ traceless matrices provides an operator realization of $A_{n}$ and a realization of the natural representation space $V\left(\omega_{1}\right)=\operatorname{span}_{\mathbb{C}}\left\{x_{1}, \ldots, x_{n+1}\right\}$.

Fix $\vec{a}=\left(a_{1}, \ldots, a_{n+1}\right) \in \mathbb{C}^{n+1}$ where none of the components are integers, and consider the space

$$
N(\vec{a})=\operatorname{span}_{\mathbb{C}}\left\{x^{\vec{b}}=x_{1}^{b_{1}} \cdots x_{n+1}^{b_{n+1}} \mid b_{i}-a_{i} \in \mathbb{Z}, \text { for all } i \text { and } \sum_{i=1}^{n+1}\left(b_{i}-a_{i}\right)=0\right\} .
$$

Using the realization of $A_{n}$ in terms of operators we easily see that $N(\vec{a})$ is a simple torsion-free $A_{n}$-module of degree 1. In fact in [BL1], it is shown that every simple torsion-free $A_{n}$-module of degree 1 is equivalent to such a module.

We now consider the tensor product module $M$ given by $M=N\left(\vec{a}-\varepsilon_{1}\right) \otimes$ $V\left(\omega_{1}\right)$, where $\vec{a}-\varepsilon_{1}=\left(a_{1}-1, \alpha_{2}, \ldots, a_{n+1}\right)$. In [BBL], it is shown that the 
tensor product of a torsion-free module of finite degree and a finite-dimensional module is torsion-free. Hence, $M$ is torsion-free.

For each $i=1, \ldots, n+1$, we define

$$
v_{i}=x_{1}^{a_{1}} \cdots x_{i}^{a_{i}-1} \cdots x_{n+1}^{a_{n+1}} \otimes x_{i} .
$$

It is easily verified that the elements $v_{i}$ span the $\Theta$ weight space of $M$ where $\boldsymbol{\Theta}\left(E_{i i}-E_{i+1 i+1}\right)=a_{i}-a_{i+1}$. By direct computation we observe that subspaces

$$
\mathscr{W}_{1}=\operatorname{span}_{\mathbb{C}}\left\{\sum_{i=1}^{n+1} a_{i} v_{i}\right\}, \quad \mathscr{W}_{0}=\operatorname{span}_{\mathbb{C}}\left\{\sum_{i=1}^{n+1} c_{i} v_{i} \mid \sum_{i=1}^{n+1} c_{i}=0\right\}
$$

of $M_{\Theta}$ are invariant under $\mathscr{A}_{\alpha}$ for all $\alpha \in \Phi$ and hence, by Lemma 6, they are $U_{0}$ submodules. This means that $M_{0}=U \mathscr{W}_{0}$ and $M_{1}=U \mathscr{W}_{1}$ are submodules of $M$ with $\left(U \mathscr{W}_{i}\right)_{\Theta}=W_{i} . M_{1}$ is isomorphic to $N(\vec{a})$.

Suppose that $M_{0}$ contains the proper nonzero simple submodule $V$ of degree $k \leq n-1$, and hence by our Main Theorem $k=1$ or $k=n-1$. Suppose that $k=n-1$. Then no vector of the form $v_{i}-v_{j}$ with $i \neq j$ is in $V$ because such vectors generate $\mathscr{W}_{0}$ and hence generate $M_{0}$ as we see from

$$
X_{i} \partial_{k} X_{k} \partial_{i}\left(v_{i}-v_{j}\right)=a_{i}\left(a_{k}+1\right)\left(v_{i}-v_{j}\right)-a_{k}\left(v_{i}-v_{k}\right) \text { for all } k \neq i, j .
$$

By a dimension argument, we know that $\left(v_{1}-v_{2}\right)-b\left(v_{1}-v_{3}\right) \in V$ for some $b \in \mathbb{C}$ and, since $-v_{2}+v_{3} \notin V$, we know that $b \neq 1$. Computing we have

$$
\begin{aligned}
& X_{1} \partial_{4} X_{4} \partial_{1}\left(\left(v_{1}-v_{2}\right)-b\left(v_{1}-v_{3}\right)\right) \\
& \quad=\left[a_{1}\left(a_{4}+1\right)\left(v_{1}-v_{2}\right)-a_{4}\left(v_{1}-v_{4}\right)\right]-b\left[a_{1}\left(a_{4}+1\right)\left(v_{1}-v_{3}\right)-a_{4}\left(v_{1}-v_{4}\right)\right],
\end{aligned}
$$

from which it follows that $v_{1}-v_{4} \in V$. Hence, $k \neq n-1$ and so $k=1$. This means that there is some $v=\sum_{i=1}^{n+1} d_{i} v_{i} \in V \cap M_{\Theta}$ with not all $d_{i}=0$ for $i \geq 3$ which is an eigenvector for every $X_{i} \partial_{k} X_{k} \partial_{i}$.

$$
\begin{aligned}
X_{1} \partial_{2} X_{2} \partial_{1} v= & \left(d_{1}\left(a_{1}-1\right)\left(a_{2}+1\right)+d_{1}+d_{2} a_{1}\right) v_{1}+\left(d_{1} a_{2}+d_{2} a_{1} a_{2}\right) v_{2} \\
& +d_{3} a_{1}\left(a_{2}+1\right) v_{3}+\cdots+d_{n+1} a_{1}\left(a_{2}+1\right) v_{n+1}
\end{aligned}
$$

tells us that the eigenvalue for this operator is $a_{1}\left(a_{2}+1\right)$, and it forces $d_{1}=s a_{1}$ and $d_{2}=s a_{2}$ for some $s \in \mathbb{C}$. Similar calculations show that $d_{i}=s a_{i}$. Therefore, $M_{0}$ is a simple torsion-free $A_{n}$-module of degree $n$ and $M=$ $M_{1} \oplus M_{0}$ unless $\sum_{i=1}^{n+1} a_{i}=0$, in which case we have a composition series $\{0\} \subset M_{1} \subset M_{0} \subset M$. When $M_{1} \subset M_{0}$, the quotient $M_{0} / M_{1}$ is a simple torsion-free $A_{n}$-module of degree $n-1$.

\section{REFERENCES}

[BBL] G. Benkart, D. J. Britten, and F. W. Lemire, On the tensor product of pointed torsion free and simple finite dimensional $A_{n}$ modules (in progress).

[BL1] D. J. Britten and F. W. Lemire, A classification of simple Lie modules having a 1-dimensional weight space, Trans. Amer. Math. Soc. 299 (1987), 687-697.

[BL2] - On basic cycles of $A_{n}, B_{n}, C_{n}$ and $D_{n}$, Canad. J. Math. XXXVII (1985), 122-140.

[BL3] Math. Soc. 272 (1982), 509-540. 
[F] Suren Fernado, Lie algebra modules with finite dimensional weight spaces. I, Trans. Amer. Math. Soc. 322 (1987), 757-781.

[J] J. Humphreys, Introduction to Lie algebras and representation theory, Graduate Texts in Math., vol. 9, Springer-Verlag, New York, 1972.

Department of Mathematics, University of Windsor, Windsor, Ontario, CANADA N9B 3P4

E-mail address: britteneserver.uwindsor.ca

E-mail address: lemirelserver.uwindsor.ca 